\title{
Tyrosine kinase inhibitors interstitial pneumonitis: diagnosis and management
}

\author{
Yayi He, Caicun Zhou \\ Department of Medical Oncology, Shanghai Pulmonary Hospital, Tongji University Medical School Cancer Institute, Tongji University School of \\ Medicine, Shanghai 200433, China \\ Correspondence to: Caicun Zhou. Department of Medical Oncology, Shanghai Pulmonary Hospital, Tongji University Medical School Cancer \\ Institute, Tongji University School of Medicine, No. 507 Zhengmin Road, Shanghai 200433, China. Email: caicunzhoudr@163.com.
}

Submitted Apr 18, 2019. Accepted for publication May 06, 2019.

doi: $10.21037 /$ tlcr.2019.05.02

View this article at: http://dx.doi.org/doi: 10.21037/tlcr.2019.05.02

Lung cancer is the most common cancer in the world (1-3). In the past 10 years, studies have shown that tyrosine kinase inhibitors (TKIs), such as gefitinib, afatinib, and crizotinib, provide significant benefits to advanced non-small cell lung cancer (NSCLC) patients $(4,5)$. NSCLC with positive epidermal growth factor receptor $(E G F R)$ or anaplastic lymphoma kinase $(A L K)$ mutations can achieve longer progression-free survival (PFS) and a higher life quality through treatment with TKIs $(4,5)$.

It should be noted that there are some side effects of TKIs, such as increased alanine aminotransferase concentrations, hepatic dysfunction, skin rash, diarrhea, etc. (5-9). Although it is uncommon, drug-induced interstitial lung disease (ILD) is one of the most serious complications of TKIs (10-12), and is thus a major concern during EGFR-TKI treatment $(11,12)$. The incidence of ILD is about $1 \%$ in TKI-treated NSCLC patients worldwide; it was reported that ILD occurred in about $3.5 \%$ of NSCLC patients treated with gefitinib in Japan (13). Meanwhile, the frequency of ILD induced by $A L K$ has been reported to be $<1 \%(14-17)$.

There are some high-risk factors for ILD in patients with TKIs: male sex, smoking, and a history of pulmonary fibrosis (18). Interstitial shadow on chest CT has been correlated with TKI-related ILD (19), and it was reported that prior radiotherapy was also a risk factor for ILD $(11,12,19,20)$. Another potential risk factor of TKI-induced ILD is Anti-PD-1 monoclonal antibodies (21).

The incidence, histopathology, pathophysiology, and prognosis of TKI-induced ILD remain poorly understood. The pathogenesis of ILD was reported to be related to different types of cytotoxicity. In this process, TKIs directly injure the endothelium of alveolar capillaries and/or the pneumocytes. Cytokines are released and the inflammatory cells are recruited. These cytokines releasing induce the dysfunction of endothelial and lung edema (22). It was reported that increasing IL-6 played an important role in EGFR-TKI-related ILD, and blocking of IL-6 pathway could reduce the incidence of ILD during TKI treatment (23). For gefitinib-induced ILD, metformin demonstrated an inhibitory effect, while EGFR-TKI combined with metformin in treating NSCLC patients was shown to reduce drug-related adverse reactions (24).

Clinical manifestations of ILD are cough, fever, and acute or subacute dyspnea, and patients with ILD usually need to be hospitalized. Respiratory failure is common, and its occurrence in one third of cases is fatal. Chest computed tomography (CT) is similar to interstitial pneumonitis, while radiographic findings are nonspecific. The histopathology of ILD induced by TKIs is diffuse alveolar damage. As it is rare that tissues can be acquired from these patients, diagnosis is often based on clinical and radiographic findings (18).

The following points can assist in the diagnosis of ILD induced by TKIs (18):

(I) Acute or subacute, progressive dyspnea with or without cough and/or fever;

(II) Exclusion of pulmonary infection;

(III) Radiographic findings showing bilateral, diffuse, or patchy interstitial and/or alveolar opacifications without evidence of marked progression of lung 
cancer;

(IV) Pathologic findings consistent with ILD (if available).

If TKI-induced ILD is diagnosed, it is important to give patients symptomatic supportive treatment. As immune disorder may be one of the mechanisms of TKI-induced ILD, it is often treated with steroids. Discontinuation of TKIs is another critical option, but can pose a clinical dilemma: some patients may receive the benefits of TKIs before ILD occurs, some patients may have no suitable alternative, and some patients may suffer severe side effects if they switch to other treatments. It was reported that TKI could continue to be used with steroids (25). Doctors need to judge the benefits and risks and closely monitor their patients (26). Some patients can recover from TKIinduced ILD through a reduction of its dosage. This might be another potential therapeutic option for treating TKI-induced ILD. Doctors need to pay attention to the recurrence of ILD during the time patients are being treated with a reduced TKI dose. Overall, the understanding of the underlying mechanisms of ILD and strategies to overcome TKI-induced ILD is immature, and further investigation is required in these areas (27).

In summary, TKIs have heralded a new era in the treatment of cancers, but ILD still remains its most serious side effects. Male sex, smoking, history of pulmonary fibrosis, history of radiotherapy, and combination with immune therapy, are the risk factors of TKI-induced ILD. TKI-induced ILD presents with cough, fever, dyspnea, and hypoxemia. Should TKI-induced ILD be diagnosed, symptomatic supportive treatment is important, and patients should be treated with steroids which can be used simultaneously with TKIs. The discontinuation of TKIs can be clinically problematic as other treatment options may not be viable, and doctors should pay close attention to these patients. The above facts notwithstanding, the mechanisms of ILD and strategies to overcome TKI-induced ILD are limited, and need continued study focus.

\section{Acknowledgments}

Funding: This study was supported in part by a grant from National Natural Science Foundation of China (81802255), Shanghai Pujiang Program (17PJD036) and a grant from Shanghai Municipal Commission of Health and Family Planning Program (20174Y0131). National Key Research \& Development Project (2016YFC0902300), Major Disease
Clinical Skills Enhancement Program of Three-year Action Plan for Promoting Clinical Skills and Clinical Innovation in Municipal Hospitals, and Shanghai Shen Kang Hospital Development Center Clinical Research Plan of SHDC (16CR1001A). The fundamental research funds for the central universities.

\section{Footnote}

Conflicts of Interest: The authors have no conflicts of interest to declare.

\section{References}

1. Camidge DR, Pao W, Sequist LV. Acquired resistance to TKIs in solid tumours: learning from lung cancer. Nat Rev Clin Oncol 2014;11:473-81.

2. Politi K, Herbst RS. Lung cancer in the era of precision medicine. Clin Cancer Res 2015;21:2213-20.

3. Siegel RL, Miller KD, Jemal A. Cancer statistics, 2018. CA Cancer J Clin 2018;68:7-30.

4. Zhou C, Wu YL, Chen G, et al. Erlotinib versus chemotherapy as first-line treatment for patients with advanced EGFR mutation-positive non-small-cell lung cancer (OPTIMAL, CTONG-0802): a multicentre, open-label, randomised, phase 3 study. Lancet Oncol 2011;12:735-42.

5. Mok TS, Wu YL, Ahn MJ, et al. Osimertinib or PlatinumPemetrexed in EGFR T790M-Positive Lung Cancer. N Engl J Med 2017;376:629-40.

6. Cameron L, Solomon B. New Treatment Options for ALK-Rearranged Non-Small Cell Lung Cancer. Curr Treat Options Oncol 2015;16:49.

7. Paz-Ares L, Mezger J, Ciuleanu TE, et al. Necitumumab plus pemetrexed and cisplatin as first-line therapy in patients with stage IV non-squamous non-small-cell lung cancer (INSPIRE): an open-label, randomised, controlled phase 3 study. Lancet Oncol 2015;16:328-37.

8. Thatcher N, Hirsch FR, Luft AV, et al. Necitumumab plus gemcitabine and cisplatin versus gemcitabine and cisplatin alone as first-line therapy in patients with stage IV squamous non-small-cell lung cancer (SQUIRE): an open-label, randomised, controlled phase 3 trial. Lancet Oncol 2015;16:763-74.

9. Zhou C, Wu YL, Chen G, et al. Final overall survival results from a randomised, phase III study of erlotinib versus chemotherapy as first-line treatment of EGFR 
mutation-positive advanced non-small-cell lung cancer (OPTIMAL, CTONG-0802). Ann Oncol 2015;26:1877-83.

10. Inoue A, Xin H, Suzuki T, et al. Suppression of surfactant protein A by an epidermal growth factor receptor tyrosine kinase inhibitor exacerbates lung inflammation. Cancer Sci 2008;99:1679-84.

11. Inoue A, Saijo Y, Maemondo M, et al. Severe acute interstitial pneumonia and gefitinib. Lancet 2003;361:137-9.

12. Hotta K, Kiura K, Tabata M, et al. Interstitial lung disease in Japanese patients with non-small cell lung cancer receiving gefitinib: an analysis of risk factors and treatment outcomes in Okayama Lung Cancer Study Group. Cancer J 2005; 11:417-24.

13. Ando M, Okamoto I, Yamamoto N, et al. Predictive factors for interstitial lung disease, antitumor response, and survival in non-small-cell lung cancer patients treated with gefitinib. J Clin Oncol 2006;24:2549-56.

14. Camidge DR, Bang YJ, Kwak EL, et al. Activity and safety of crizotinib in patients with ALK-positive non-small-cell lung cancer: updated results from a phase 1 study. Lancet Oncol 2012;13:1011-9.

15. Dy GK, Adjei AA. Understanding, recognizing, and managing toxicities of targeted anticancer therapies. CA Cancer J Clin 2013;63:249-79.

16. Shaw AT, Kim DW, Nakagawa K, et al. Crizotinib versus chemotherapy in advanced ALK-positive lung cancer. $\mathrm{N}$ Engl J Med 2013;368:2385-94.

17. van Geel RM, Hendrikx JJ, Vahl JE, et al. Crizotinibinduced fatal fulminant liver failure. Lung Cancer 2016;93:17-9.

18. Yoneda KY, Shelton DK, Beckett LA, et al. Independent review of interstitial lung disease associated with death in TRIBUTE (paclitaxel and carboplatin with or without concurrent erlotinib) in advanced non-small cell lung cancer. J Thorac Oncol 2007;2:537-43.

Cite this article as: $\mathrm{He} \mathrm{Y,} \mathrm{Zhou} \mathrm{C.} \mathrm{Tyrosine} \mathrm{kinase} \mathrm{inhibitors}$ interstitial pneumonitis: diagnosis and management. Transl Lung Cancer Res 2019;8(Suppl 3):S318-S320. doi: 10.21037/ tlcr.2019.05.02
19. Niho S, Goto K, Yoh K, et al. Interstitial shadow on chest CT is associated with the onset of interstitial lung disease caused by chemotherapeutic drugs. Jpn J Clin Oncol 2006;36:269-73.

20. Chiang CL, Chen YW, Wu MH, et al. Radiation recall pneumonitis induced by epidermal growth factor receptortyrosine kinase inhibitor in patients with advanced nonsmall-cell lung cancer. J Chin Med Assoc 2016;79:248-55.

21. Mamesaya N, Kenmotsu H, Katsumata M, et al. Osimertinib-induced interstitial lung disease after treatment with anti-PD1 antibody. Invest New Drugs 2017;35:105-7.

22. Vahid B, Marik PE. Pulmonary complications of novel antineoplastic agents for solid tumors. Chest 2008;133:528-38.

23. Ishiguro $Y$, Ishiguro H, Miyamoto H. Epidermal growth factor receptor tyrosine kinase inhibition up-regulates interleukin-6 in cancer cells and induces subsequent development of interstitial pneumonia. Oncotarget 2013;4:550-9.

24. Li L, Huang W, Li K, et al. Metformin attenuates gefitinib-induced exacerbation of pulmonary fibrosis by inhibition of TGF-beta signaling pathway. Oncotarget 2015;6:43605-19.

25. Kashiwabara K, Semba H, Fujii S, et al. Outcome in advanced non-small cell lung cancer patients with successful rechallenge after recovery from epidermal growth factor receptor tyrosine kinase inhibitor-induced interstitial lung disease. Cancer Chemother Pharmacol 2017;79:705-10.

26. Shah RR. Tyrosine Kinase Inhibitor-Induced Interstitial Lung Disease: Clinical Features, Diagnostic Challenges, and Therapeutic Dilemmas. Drug Saf 2016;39:1073-91.

27. Tian Q, Chen LA. Erlotinib achieved partial response in a non-small cell lung cancer patient with gefitinib-induced interstitial lung disease. Case Rep Oncol 2011;4:464-6. 